\title{
La sostenibilidad y las clases online en la universidad en tiempos de COVID-19: ¿Nos ha servido como punto de partida para una nueva modalidad de enseñanza?
}

\section{Sustainability and online classes at the university in the times of COVID-19: Has it served us as a starting point for a new kind of teaching?}

\author{
FERNÁNDEZ FERNÁNDEZ, María ${ }^{1}$ \\ MARTÍNEZ-NAVALON , Juan $\mathrm{G}^{2}$ \\ GELASHVILI, Vera ${ }^{3}$
}

\begin{abstract}
Resumen
El estudio, marcado por la pandemia por COVID-19, investigó cómo afectó a los estudiantes universitarios el cambio de enseñanza presencial a modalidad online y cómo afectó a la sostenibilidad de sus universidades. A través de un cuestionario online se recogió una muestra de 411 encuestados, que fue posteriormente validado y analizado a través del programa informático SPSS. Los resultados remarcan la importancia de asegurarles una buena educación y cuidar su bienestar para garantizará el éxito de la sociedad.
\end{abstract}

Palabras clave: estudiantes, enseñanza online, COVID-19, sostenibilidad.

\begin{abstract}
The investigation, marked by the COVID-19 pandemic, researched how the change from face-to-face to online teaching affected university students and how it affected the sustainability of their universities. An online questionnaire was used to collect a sample of 411 participants, which was then validated and analyzed through the SPSS software. The results highlight the importance of ensuring a good education and caring for their welfare to ensure the success of society Key words: students, online education, COVID-19, sustainability.
\end{abstract}

\section{Introducción}

El presente trabajo de investigación se enmarca en un contexto de crisis, incertidumbre y cambios marcados por la enfermedad del virus COVID-19. Son incuestionables las dimensiones de esta crisis y la cantidad de ámbitos a los que ha afectado, sin embargo, esta investigación se centrará en el sector de la educación superior y la sostenibilidad.

La razón del tema de este estudio radica en la importancia de cuidar de las generaciones futuras, en las que un pilar fundamental de la sociedad presente y futura, lo forman los estudiantes. Es por ello que, este estudio de

\footnotetext{
${ }^{1}$ Master en Alta Dirección. Universidad Rey Juan Carlos. m.fernandezfernandez111@alumnos.urjc.es

2 Profesor ayudante doctor. Departamento Economía de la Empresa. Universidad Rey Juan Carlos. juangabriel.martinez@urjc.es

${ }_{3}^{3}$ Profesora ayudante doctor. Departamento Economía de la Empresa. Universidad Rey Juan Carlos. vera.gelashvili@urjc.es
} 
investigación tiene la finalidad de analizar los efectos que el COVID-19 ha provocado en los estudiantes universitarios y en la sostenibilidad de sus universidades (García-Peñalvo, Corell, Abella-García, y Grande, 2020), y averiguar si este cambio de la docencia presencial a la online, sirve de base para una nueva modalidad de enseñanza en el futuro o si esta solución es sostenible en el tiempo.

El término sostenibilidad, es un concepto a la orden de día en las agendas políticas y enormemente estudiado por una gran cantidad de autores. Además, aunque posee varias formas de clasificar sus dimensiones, en esta investigación se estudiará la clasificación más utilizada: sostenibilidad social, medioambiental y económica (Asche et al., 2018). Se pueden definir las tres dimensiones de la sostenibilidad de la siguiente manera: por un lado, la sostenibilidad medioambiental sería aquella en la que los ecosistemas mantienen sus características fundamentales de forma indefinida a través del tiempo; por otro lado, la sostenibilidad económica sería aquella en la que se produce una rentabilidad razonable y estable en el tiempo; y por último, la sostenibilidad social es en la que en el que el manejo y organización sean compatibles con los valores culturales y éticos del grupo y de la sociedad (Ukko, Saunila, Rantala, y Havukainen, 2019).

Es importante tener en cuenta que según el término de sostenibilidad ha ido extendiéndose, las universidades han ido incorporando gradualmente el concepto de desarrollo sostenible a la realidad cotidiana de sus facultades (Larrán-Jorge y Andrades-Peña, 2015). Sin embargo, aunque la mayor parte de las universidades han firmado declaraciones y compromisos de sostenibilidad en educación, únicamente algunas de ellas incorporan actividades docentes relacionadas con la sostenibilidad (Huerta-Riveros y Gaete-Feres, 2017).

Es en este contexto cuando aparece la crisis del COVID-19 para desestabilizar todos los sectores de la sociedad desencadenando consecuencias sociales, económicas, laborales y medioambientales, entre las que se encuentra el objeto principal de estudio de esta investigación, la educación superior y los estudiantes universitarios. Una de las medidas optadas para mitigar el contagio fue el llamado confinamiento, que obligó a permanecer en casa a casi toda la población mundial, y a comenzar a trabajar y a estudiar desde casa. De esta manera, la enseñanza pasó de ser presencial a ser forzosamente online y puso en evidencia y amplificó la aparición de brechas sociales, tecnológicas, económicas y competenciales (García-Peñalvo et al., 2020).

Para llevar a cabo el estudio y responder a las preguntas objeto de la investigación, se llevó a cabo la elaboración de un cuestionario online que se difundió mediante redes sociales (Instagram, Twitter, Facebook, WhatsApp) y correo electrónico. El cuestionario, cuya muestra es de 411 respuestas, se dividió en cuatro bloques: clasificación del estudiante, impacto del COVID-19, sostenibilidad y clases online, y se utilizó una escala de Likert para medir el sentimiento de los encuestados. Una vez recogida la muestra, se llevó a cabo un análisis de fiabilidad con el programa informático SPSS, que validó el cuestionario mediante el índice KMO y prueba de esfericidad de Bartlett y confirmó su fiabilidad.

Una vez analizados los resultados, se cocnluye que el COVID-19 ha afectado de manera negativa a la vida de los estudiantes universitarios del país y aunque es sostenible medioambientalmente hablando; social y económicamente no lo ha sido. Sin embargo, a pesar de las circunstancias, es un cambio que ha salido adelante y ha terminado un curso que parecía que podía cerrarse en marzo. Por lo tanto, se puede llegar a la conclusión de que puede llegar a ser un cambio sostenible si se dirige bien, se reflexiona sobre los aspectos que han funcionado y los que no, y si se evalúa la mejor manera de llevarlo a cabo.

Para llevar a cabo este estudio se comenzará con una descripción bibliográfica de los conceptos más importantes que abarcan la investigación. A continuación, se describirá la metodología utilizada, y se expondrán y analizarán los resultados del mismo. Por último, se comentarán las conclusiones obtenidas de la investigación. 


\section{Marco teórico}

\subsection{Sostenibilidad}

El concepto sostenibilidad aunque se origina en el verbo francés soutenir (Brown, Hanson, Liverman, y Merideth, 1987) de principios del siglo XVIII (Geissdoerfer, Savaget, Bocken, y Hultink, 2019), surgió en 1960 a raíz de la preocupación por la degradación ambiental. Fue en 1987, cuando en el Informe Brundtland se definió por primera vez el concepto de desarrollo sostenible (World Comission on Environment and Development, 1987) cuya importancia ha ido creciendo a lo largo de los años (Karji, Woldesenbet, Khanzadi, y Tafazzoli, 2019).

A pesar de la fama del concepto, se ha criticado en muchas ocasiones la definición de Brundtland de 1987 y los objetivos del desarrollo sostenible en su conjunto (Christen y Schmidt, 2012), aunque eso no ha impedido institucionalizarlo en las agendas políticas y en las estrategias de las grandes organizaciones, ya que el concepto demuestra ser un término político tan persistente como lo son la democracia, la justicia y la libertad (Geissdoerfer et al., 2019).

El punto clave del término sostenibilidad nace con la expresión 'triple objetivo' desarrollada por el ecologista y economista John Elkington (1997) que defiende que no es posible alcanzar la sostenibilidad ecológica, social o económica por separado (Esnaashari y Sarvari, 2020). Dicho esto, aunque el termino tiene diferentes dimensiones, este estudio va a centrarse en las tres más importantes y tradicionales: ser económicamente viable, ser sociablemente beneficiosa y ser ambientalmente responsable simultáneamente. Por lo tanto, se puede concluir diciendo que, es del conjunto de estas tres corrientes donde surge el término sostenibilidad (Ben Purvis, Mao, y Robinson, 2019).

\subsubsection{Sostenibilidad social}

Como se ha comentado, la sostenibilidad social es un componente crucial de la sostenibilidad global. Es una condición positiva marcada por un fuerte sentido de cohesión social e igualdad de acceso a servicios como la salud, la educación, el transporte, la vivienda y el ocio (Rogge, Theesfeld, y Strassner, 2018). Es la capacidad de la sociedad en sí misma para sostenerse y reproducirse por sí sola adecuadamente.

A pesar del reciente enfoque de la política europea en sociedades sostenibles y cohesión social, ha habido poca discusión teórica sobre la definición de la sostenibilidad social. Existe una literatura razonablemente escasa que se enfoca específicamente en la sostenibilidad social, mientras que hay otra algo más amplia sobre los términos de capital social, cohesión social (Eizenberg y Jabareen, 2017) inclusión (Thabiso y Awuzie, 2017) y exclusión social (Dempsey, Bramley, Power, y Brown, 2011).

\subsubsection{Sostenibilidad medioambiental}

La definición medioambiental de la sostenibilidad se centra en los procesos biológicos naturales y en la continua productividad y funcionamiento de los ecosistemas a lo largo del tiempo ( Purvis et al., 2019).

El concepto propiamente dicho fue probablemente diseñado por primera vez por los científicos del Banco Mundial. Originalmente, el término "ambientalmente responsable" se utilizó para describir el término "desarrollo". Posteriormente, se empleó el "desarrollo ambientalmente sostenible" y finalmente, se desarrolló el concepto de sostenibilidad ambiental (Goodland, 1998) que busca mejorar el bienestar humano protegiendo y conservando las fuentes de materias primas utilizadas para las necesidades humanas, a fin de evitar daños a los seres humanos (Hák, Janouškova, Moldan, y Dahl, 2018; Moldan, Janoušková, y Hák, 2012). 


\subsubsection{Sostenibilidad económica}

La definición de la sostenibilidad económica es más escurridiza. Los economistas tienden a encargarse del crecimiento económico y no se ocupan, en su mayor parte, del tema de la sostenibilidad (Brown et al., 1987). Sin embargo, es por esta misma razón, que la economía necesita usar la anticipación y la precaución continuamente y doblegarse necesariamente hacia el lado de la sostenibilidad (Janoušková, Hák, y Moldan, 2018). Dicho esto, podría describirse la sostenibilidad económica como el proceso de atribuir y preservar los recursos escasos, asegurando al mismo tiempo resultados sociales y ambientales positivos (Cadil, Mirosnik, Petkovova, y Mirvald, 2018). En consecuencia, las empresas que sean capaces de gestionar eficazmente su medio ambiente y la justicia social también contribuirán directamente a hacerse económicamente sostenibles (Pena-Cerezo, Artaraz-Minon, y Tejedor-Nunez, 2019).

\subsubsection{La sostenibilidad en la universidad}

Aunque al principio la sostenibilidad estaba principalmente vinculada a cuestiones medioambientales y en el área de las administraciones públicas y en la universitaria especialmente, ejercía un segundo plano (Larrán-Jorge y Andrades-Peña, 2015); según el término de sostenibilidad ha ido extendiéndose (Del Sordo, Farneti, Guthrie, Pazzi, y Siboni, 2016), las universidades han ido incorporando gradualmente el concepto de desarrollo sostenible a la realidad cotidiana de sus facultades (Lozano et al., 2015).

Sin embargo, aunque la mayor parte de las organizaciones han firmado declaraciones (Muñoz, Callejo, Sastre, y Marín, 2017) y compromisos de sostenibilidad en la educación, únicamente algunas de ellas incorporan actividades docentes relacionadas con la sostenibilidad (Huerta-Riveros y Gaete-Feres, 2017). Hoy por hoy, la sostenibilidad implica relacionar de forma ecuánime las metas económicas, sociales y medioambientales de una sociedad en vista del efecto a largo plazo de las actividades actuales.

\subsection{El COVID-19}

EI COVID-19 es una enfermedad transmisible que ha controlado los titulares desde su aparición en diciembre de 2019 en Wuhan (China). El virus produce la enfermedad infecciosa del conocido COVID-19 (acrónimo de "Coronavirus disease 2019») que, aunque en mayoría de los casos produce síntomas leves, en otros casos la enfermedad puede ser muy grave e incluso mortal (Sierra, 2020). La propagación del virus aumentó de forma exponencial el número de infecciones y fallecimientos en casi todas las zonas geográficas del mundo y ocasionó dificultades en los sistemas sanitarios de la mayoría países.

Dada la gravedad de la situación, el 11 de marzo de 2020 la Organización Mundial de la Salud (OMS) declaró el COVID-19 como pandemia mundial puesto que afectó a todo el planeta (Niranjans, 2020) causando graves consecuencias y desencadenando una profunda crisis económica y social mundial.

\subsubsection{Consecuencias socioeconómicas del COVID-19}

Las medidas adoptadas para disminuir la propagación del virus condujeron a la mayoría de los países a una parálisis de ámbitos importantes de la actividad y, por tanto, a un descenso profundo y abrupto del PIB (Banco de España, 2020). En especial, la velocidad del número de contagios llevó al Gobierno de España a aprobar un Real Decreto (RD 463/2020, de 14 de marzo) por el que se declaró el estado de alarma cuyo objetivo era proteger la salud de la ciudadanía, contener el avance la de la enfermedad y fortalecer el sistema de salud pública (Nicola et al., 2020).

La repercusión de la disminución de la actividad productiva se ha reflejado, en particular, en el mercado de trabajo. Además, como consecuencia de la paralización de sectores de actividad, un gran número de trabajadores 
sufrió el comúnmente conocido ERTE o expedientes de regulación temporal de empleo (de Las Heras-Pedrosa, Sánchez-Núñez, y Peláez, 2020).

Esta alteración de la actividad y la pérdida de puestos de trabajo han provocado una notable disminución de la demanda interna. Por otro lado, el descenso de los intercambios comerciales ha sido también muy notable y en cuanto a los servicios turísticos, el cese ha sido en algunos casos casi completo, en una situación de bloqueo de las fronteras a los movimientos de personas y desconexión del tráfico aéreo (Nicola et al., 2020).

\subsubsection{Consecuencias medioambientales del COVID-19}

Es incuestionable que el brote de COVID-19 ha implicado una respuesta sanitaria necesaria, pero la gobernanza de esta crisis sanitaria y social ha provocado una situación de inseguridad y desconcierto extremo que se ha visto reflejado en infinidad de aspectos.

Esta nueva situación ha alterado el orden de preferencias en la agenda pública de los gobiernos (Leal, Brandli, Lange, Rayman-Bacchus, y Platje, 2020) que ha obligado a los gobiernos de todos los países y al organismo climático de la ONU a aplazar la próxima Cumbre del Clima (COP26), prevista inicialmente para finales de este año, hasta una fecha aún sin concretar en el 2021.

Por su parte, la ralentización de las economías está causando una reducción temporal de las emisiones de gases de efecto invernadero y de la contaminación del aire, lo que podría inducir a un menor aliciente para luchar contra los efectos dañinos del cambio climático (Lal et al., 2020). Sin embargo, la reconstrucción económica debería ir de la mano de la sostenibilidad ya que si no, se correrá el riesgo de repetir estos errores cuando se tengan que hacer frente a los riesgos del cambio climático (Bosoer y Turzi, 2020).

\subsection{El teletrabajo}

El salto tecnológico durante el transcurso de este siglo, ha dado paso a un concepto que hasta hace cuatro décadas parecía imposible: descentralizar las actividades y tareas de las empresas (Belzunegui-Eraso y ErroGarcés, 2020). Una solución llamada "teletrabajo" que, aunque nació en tiempos de crisis, es la modalidad que pretende reconvertir a los trabajadores de la sociedad industrial en trabajadores de la sociedad de la información (Nakrošienè, Bučiūnienè, y Goštautaitè, 2019).

De hecho, el concepto nació hace más de 40 años, de la mano del físico Jack Nilles, (Messenger y Gschwind, 2016), concepto pionero y profético en una época en la que las tecnologías de información y comunicación no estaban tan desarrolladas como lo están ahora y en menos de veinte años, se ha convertido en una política tanto estatal como empresarial que sitúa a la Unión Europea a la vanguardia de la carrera tecnológica mundial (Narayanan, Menon, Plaisent, y Bernard, 2017).

En cuanto al aspecto medioambiental, no existen hoy en día excesivos estudios empíricos sobre el ahorro de energía o la reducción de la contaminación causada por la puesta en marcha del teletrabajo como medida laboral (Moos, Andrey, y Johnson, 2017). No obstante, se puede afirmar que guardan una gran afinidad. De manera que, cuanto más éxito tenga el modelo del teletrabajo, más aportará a la sostenibilidad de dicha empresa, de igual forma que si no hay una buena adaptación del modelo, empeorará la sostenibilidad de la organización y así, la de la sociedad (Belzunegui-Eraso y Erro-Garcés, 2020).

Por ello, es importante tener en cuenta que el teletrabajo no es apto para todas las empresas, no es apto para cualquier trabajador en cualquier puesto de trabajo, no en todas las ocasiones es productivo, no en todas las ocasiones supone un ahorro, y no es de por sí sostenible. Para que la adaptación de este modelo sea exitosa se deben llevar a cabo la llamada "gestión sostenible de los recursos humanos", que consiste en provisionar, organizar, retener, evaluar y desarrollar a los trabajadores (Dima, Țuclea, Vrânceanu, y Țigu, 2019). 


\subsubsection{El teletrabajo en educación: las clases online en tiempos de COVID-19}

Como se ha comentado, el teletrabajo no solo afectó a los trabajadores sino también a los estudiantes en modalidad de clases online (García-Peñalvo et al., 2020), ya que una de las primeras medidas contra la propagación del virus fue, sin duda, el cierre de los centros educativos de todos los niveles (Auger et al., 2020)

En la educación superior, objeto principal de esta investigación, ese brinco a la enseñanza a distancia provocado por la pandemia por COVID-19, supone un nivel de capacidades y destrezas digitales que no todos los profesores y estudiantes tenían tras llevar años y años habituados a un sistema presencial. Por lo tanto, no podía esperarse que esta acción, de emergencia e inesperada, fuese comparable a las que están expresamente para impartirse online (Hodges, Moore, Lockee, Trust, y Bond, 2020). Esta medida la han tenido que llevar a cabo los profesores y estudiantes sin previo aviso ni preparación alguna (García-Peñalvo et al., 2020) y ha puesto en evidencia y ha amplificado la aparición de varias brechas: acceso, uso, capacidades y competencias profesionales.

Por lo tanto, en la situación actual, es importante reflexionar concretamente sobre qué obstaculiza al sistema educativo para poder hacer frente a este nuevo cambio social de una forma más eficiente. Al final, será de los ciudadanos como sociedad de quien dependerá si se toma este acontecimiento insólito como una oportunidad vacía y desperdiciada o como una transición plena y esperanzadora hacia la tan anhelada pero evasiva sostenibilidad (Sandín, Valiente, García-Escalera, y Chorot, 2020).

\section{Metodología}

\subsection{Recogida de datos y muestra}

Para llevar a cabo este estudio se llevó a cabo la distribución de un cuestionario online autoadministrado que recogió durante el mes de junio y julio de 2020, una muestra de 411 personas. El cuestionario se difundió por redes sociales (Instagram, Facebook, Twitter y Whatsapp) y por correo electrónico, y abarcó las principales comunidades autónomas del país, lo que permitió una muestra más heterogénea de la población de estudiantes en universidades españolas (Martínez-Navalón, Gelashvili, y Debasa, 2019).

Para llevarlo a cabo, se utilizó un cuestionario de cinco puntos de escala de Likert, puesto que son los más recomendados por la facilidad de respuesta por parte del encuestado y la posibilidad de medir el sentimiento del mismo, lo que permite llevar a cabo un estudio más amplio (Martínez-Navalón et al., 2019).

\subsection{Estudio descriptivo de las variables}

El cuestionario se dividió en 4 bloques: el primero incluía la clasificación del estudiante, el segundo se centró en el impacto del COVID-19, el tercero en la sostenibilidad y el cuarto investigó el impacto de las clases online en sus diferentes dimensiones.

A continuación, se muestra un cuadro descriptivo con todas las posibles respuestas del cuestionario, su correspondiente pregunta y su equivalencia en valores cuantitativos. De esta manera, el Cuadro 1 expone la equivalencia de las respuestas a lo largo del cuestionario, es decir, cada número representa los valores cualitativos que se han utilizado para medir la validez del cuestionario y las respuestas representan los valores cuantitativos que corresponden al valor numérico de cada pregunta. 
Cuadro 1

Cuadro de equivalencia de las variables

\begin{tabular}{|c|c|c|c|c|c|}
\hline 1 & 2 & 3 & 4 & 5 & № pregunta \\
\hline $\begin{array}{l}\text { Muy en } \\
\text { desacuerdo }\end{array}$ & En desacuerdo & $\begin{array}{l}\text { Ni de acuerdo ni en } \\
\text { desacuerdo }\end{array}$ & De acuerdo & $\begin{array}{l}\text { Muy de } \\
\text { acuerdo }\end{array}$ & $\begin{array}{c}5-7,17-28,32-34 \\
44-46,50-55\end{array}$ \\
\hline Sí & No & & & & 9-10, \\
\hline Sí, confirmado & Sí, se sospecha & No, no se sospecha & & & $11-12$ \\
\hline Nada & Un poco & Bastante & Mucho & & 13, 41-43, \\
\hline Muy malo & Malo & Normal & Bueno & Muy bueno & 14 \\
\hline $\begin{array}{l}\text { No suspendo } \\
\text { nunca }\end{array}$ & $\begin{array}{l}\text { Casi nunca } \\
\text { suspendo }\end{array}$ & $\begin{array}{l}\text { Suelo suspender } \\
\text { alguna }\end{array}$ & $\begin{array}{l}\text { Siempre } \\
\text { suspendo }\end{array}$ & & 15 \\
\hline Solo & $\begin{array}{l}\text { Con mi pareja e } \\
\text { hijos }\end{array}$ & $\begin{array}{l}\text { En una residencia } \\
\text { universitaria }\end{array}$ & $\begin{array}{l}\text { Con mis padres } \\
\text { y/o familiares }\end{array}$ & & 8 \\
\hline Nunca & Pocas veces & Algunas veces & Con frecuencia & Siempre & $\begin{array}{c}29-31,35-40,47- \\
49\end{array}$ \\
\hline Presencialmente & En casa & $\begin{array}{l}\text { Combinando ambas } \\
\text { modalidades }\end{array}$ & & & 56 \\
\hline $\begin{array}{l}\text { Menor de } 18 \\
\text { años }\end{array}$ & $\begin{array}{l}\text { Entre } 18 \text { y } 24 \\
\text { años }\end{array}$ & Entre 24 y 26 años & $\begin{array}{l}\text { Mayor de } 40 \\
\text { años }\end{array}$ & & 2 \\
\hline Hombre & Mujer & Otros & & & 1 \\
\hline URJC & UCM & UPM & UC3M & Otros & 3 \\
\hline
\end{tabular}

Para simplificar también, el posterior análisis de los resultados, se procedió a reducir todas las preguntas mediante el uso de abreviaturas. El cuadro Anexo 1. de equivalencia: variable-pregunta, que se encuentra en los Anexos, recopila todas las preguntas objeto de esta investigación con su correspondiente abreviatura.

\subsection{Estudio descriptivo de los métodos de validación y análisis}

Una vez obtenida la versión reducida del cuestionario se analizaron las diferentes relaciones entre los factores obtenidos tras el análisis y se realizó la validación del cuestionario mediante el formato SPPS.

SPSS es un paquete estadístico diseñado por IBM Corporation y ampliamente utilizado por investigadores y académicos de todo el mundo. Se caracteriza por ser muy fácil de usar y ser capaz de realizar una gran variedad de pruebas estadísticas entre las que se encuentra el análisis de confiabilidad y validez de un cuestionario (Ong y Puteh, 2017), lo que justifica la elección del paquete informático para esta investigación.

El análisis de fiabilidad llevado a cabo con SPSS se estableció mediante el coeficiente alfa de Cronbach, basándose en la correlación de elementos y variables medidos en escala de Likert. Los valores del coeficiente alfa de Cronbach propuestos por Nunnally y Bernstein fijaron un umbral mínimo de 0,7 (Reyes-Menendez, Saura, y Martinez-Navalon, 2019) dentro de un rango entre 0 y 1 , de manera que se consideraría un coeficiente aceptable cuando su valor es igual o superior a 0,7 (Bland y Altman, 1997;Dagiliūtè, Liobikienè, y Minelgaitè, 2018).

Además del Alpha de Cronbach, otro indicador de la fiabilidad y validez de un cuestionario es el índice KMO, Medida de Adecuación de la Muestra Kaiser, Meyer y Olkin, que se encarga de medir la correlación e interrelación existente entre las variables (Isman y Canan, 2014). Para interpretarlo se debe tener en cuenta que en un rango de 0 a 1, un valor igual o superior a 0,7 indica una interrelación satisfactoria de los elementos. Cabe destacar, 
también, la prueba de esfericidad de Bartlett, que evalúa la aplicabilidad del análisis factorial de las variables estudiadas en el caso en el que el Sig. (p-valor) sea menor a 0,05 (Howard, 2016).

\section{Resultados}

\subsection{Validez y fiabilidad de la escala de medida}

En este estudio, el alfa de Cronbach salió muy justo a 0,7 por la cantidad de variables estudiadas (54), por lo que no resultaba significativo y se decidió examinar la fiabilidad de la encuesta también aplicando el índice KMO. Como se puede ver en el siguiente cuadro, el resultado del test de fiabilidad presenta un índice KMO de 0,81 y la prueba de Bartlett muestra un sig. menor a 0’05, que resulta adecuado para confirmar la fiabilidad de la encuesta.

\section{Cuadro 2}

Prueba de KMO y Bartlett

\begin{tabular}{llr}
\hline Medida de Kaiser-Olkin de adecuación de muestreo & $\mathbf{0 , 8 1 0}$ \\
\hline \multirow{2}{*}{$\begin{array}{l}\text { Prueba de esfericidad de } \\
\text { Bartlett }\end{array}$} & Aprox. Chi-cuadrado & 7227,063 \\
\cline { 2 - 3 } & gl & 1431 \\
\cline { 2 - 3 } & Sig. & 0,000 \\
\hline
\end{tabular}

Fuente de elaboración propia

\subsection{Análisis de los resultados del cuestionario}

Teniendo en cuenta el cuadro que se muestra a continuación, se puede apreciar que casi un $70 \%$ de la encuesta ha sido realizada por mujeres y un $85 \%$ del conjunto de los estudiantes tiene entre 18 y 26 años. Además, cabe destacar que el $50 \%$ de la muestra proviene de la Universidad Rey Juan Carlos y más de un $70 \%$ es procedente de universidades de la Comunidad de Madrid. Sin embargo, la encuesta ha tenido amplio alcance por el país obteniendo respuestas, además de Madrid, en Cataluña, Comunidad Valenciana, Aragón, Andalucía, Castilla La Mancha, Castilla y León, Galicia, Asturias y las Islas Canarias.

Cuadro 3

Resultados del primer bloque: Clasificación del estudiante

\begin{tabular}{|c|c|c|c|}
\hline CLASIFICACIÓN & VARIABLE & FRECUENCIA & PORCENTAJE \\
\hline \multirow{3}{*}{ Género } & Hombre & 128 & $31 \%$ \\
\hline & Mujer & 278 & $68 \%$ \\
\hline & Otros & 5 & $1 \%$ \\
\hline \multirow{5}{*}{ Edad } & Menor de 18 años & 7 & $2 \%$ \\
\hline & Entre 18 y 24 años & 351 & $85 \%$ \\
\hline & Entre 24 y 26 años & 29 & $7 \%$ \\
\hline & Entre 26 y 40 años & 13 & $3 \%$ \\
\hline & Mayor de 40 años & 11 & $3 \%$ \\
\hline \multirow{5}{*}{ Universidad } & URJC & 207 & $50 \%$ \\
\hline & UCM & 40 & $10 \%$ \\
\hline & UPM & 20 & $5 \%$ \\
\hline & UC3M & 18 & $4 \%$ \\
\hline & OTROS & 126 & $31 \%$ \\
\hline
\end{tabular}


Por otro lado, en el Cuadro anexo 2. Resultados de los bloques 2, 3 y 4, que se encuentra en los Anexos, se pueden observar los resultados de los bloques restantes de la encuesta: COVID-19, sostenibilidad y clases online, de los que van a mencionarse algunos de los más relevantes.

En primer lugar, se puede mencionar que el $65 \%$ de los estudiantes se ha sentido menos capaz de realizar sus tareas y un $62 \%$ ha encontrado dificultades para conciliar su vida familiar con su vida estudiantil. Resaltando que un $87 \%$ de ellos ha vivido con sus familiares durante este periodo de confinamiento por COVID-19, aunque solamente un $31 \%$ ha vivido con niños menores de 18 años en casa. Dentro de este grupo, el $41 \%$ de los estudiantes está de acuerdo y muy de acuerdo en que sus relaciones familiares han empeorado en este periodo y solo un $20 \%$ considera que mejorarían si continuase en el futuro este método de enseñanza. Por otro lado, cabe mencionar que el $77 \%$ de la muestra tiene la certeza de no ha padecido la enfermedad y un $70 \%$ está seguro de que sus familiares tampoco.

En cuanto a las herramientas tecnológicas y espacio de estudio, un $60 \%$ se ha adaptado bien al manejo de las nuevas tecnologías y un 93\% está de acuerdo o muy de acuerdo en que el acceso al aula virtual de su universidad ha sido pleno. Es importante resaltar también que el $90 \%$ ha tenido siempre o casi siempre acceso a un ordenador propio, el $81 \%$ ha tenido espacio individual y privado para el estudio siempre o casi siempre y el $92 \%$ buena conexión a internet y wifi.

Con respecto a la carga de trabajo, se puede mencionar que un $66 \%$ considera que se distrae más con el formato online y un $64 \%$ se ha sentido presionado por la carga de trabajo de los profesores. Además, el $73 \%$ está de acuerdo o muy de acuerdo con que es necesario ir a la universidad. Por otro lado, un $66 \%$ conoce las ventajas de las clases online, aunque solo un $31 \%$ está de acuerdo o muy de acuerdo en conocer los aspectos legales de las mismas.

En cuanto a las preguntas relacionadas con la sostenibilidad, es importante recalcar que de las cinco posibles respuestas, la opción más significativa, agrupando casi la mitad de la muestra, ha marcado la opción de "Ni en desacuerdo ni de acuerdo", por lo que se puede entender que la gran parte desconoce las labores de su universidad en aspectos de sostenibilidad y tampoco tiene clara su opinión al respecto.

Por último, cabe mencionar la pregunta final del cuestionario, en la que se muestra un $52 \%$ de respuestas a favor de la modalidad presencial, un $41 \%$ que prefiere ambas modalidades quedando el $7 \%$ apoyando la modalidad online.

\section{Conclusiones}

Tanto la investigación llevada a cabo como la revisión de la literatura, han posibilitado afirmar que la situación acontecida y el brusco cambio que se ha sufrido como consecuencia de la pandemia ha generado un ambiente de incertidumbre que ha afectado a la salud emocional de los estudiantes y se ha visto reflejado en su rendimiento.

Las universidades españolas tenían preparadas sus clases de manera presencial y este tipo de modificaciones necesitan tiempo, estudios y evaluaciones previas, por lo que el resultado concluye en que el cambio no se ha realizado como se debía y aunque es sostenible medioambientalmente hablando, social y económicamente no lo ha sido. No obstante, podría considerarse un milagro que con estas características y en este contexto extremo, los resultados hubiesen sido óptimos.

Por otro lado, gracias a este estudio se ha podido observar que casi el total de los estudiantes disponían de internet, ordenador propio y una gran parte no había tenido problema para adaptarse a la nueva tecnología 
docente. Esto se debe a que la mayor parte de los estudiantes son jóvenes y se han criado en el mundo de la tecnología. Por lo tanto, es una generación que ha nacido para ello. ¿Era este el momento que se necesitaba?

Está claro que nadie había planificado su actuación ante una situación de emergencia como ésta debido a la escasa probabilidad de que ocurriese. Sin embargo, esta crisis debería ser un antes y un después en el mundo para que los países prevengan escenarios futuros y desarrollen planes de actuación, en los que, en el caso de la educación, la docencia online se presenta como una medida que asegura la salud de los estudiantes y que garantiza la continuidad de la educación.

Es importante recalcar que es una oportunidad única para mejorar la educación y la economía yendo necesariamente de la mano de la lucha contra los problemas climáticos. Es decir, es una oportunidad única para la sostenibilidad. La pandemia ha puesto a la humanidad a prueba, ha sido como un experimento espontáneo que ha hecho inevitablemente necesario replantear la vida e imaginar una nueva manera de enfrentarse a las más simples rutinas.

Como decía Albert Einstein "en los momentos de crisis, solo la imaginación es más importante que el conocimiento". Es momento de reconstruir, rediseñar y reimaginar la educación que formará parte del futuro. Se ha reflejado que el cambio no ha sido bien recibido, pero puede llegar a serlo si se prepara de otra manera. Se habrá perdido una gran oportunidad si en sectores como la educación, las universidades no se paran a reflexionar sobre la situación vivida,y no se analiza qué cosas se pueden mantener de la nueva situación. Como cita el autor Berlot Brencht (1997, p. 290), (Brencht, 1997) "la crisis se produce cuando lo viejo no acaba de morir y lo nuevo no acaba de nacer". ¿El sistema educativo mundial necesitaba renovarse? ¿Era sostenible? Es momento de cambiar.

La educación superior es el último paso que define y marca la futura línea de trabajadores puesto que serán ellos los que formen parte de las futuras empresas y de la futura sociedad. Preocuparse del bienestar, preparación profesional y desarrollo de competencias de los estudiantes, garantizará el éxito de la sociedad en la que vivan, ya que, para poder satisfacer las necesidades de los estudiantes en el futuro, hay que preocuparse por su progreso en el presente. Y como decía Miguel de Unamuno, "el verdadero progreso consiste en renovarse".

\section{Referencias bibliográficas}

Asche, F., Garlock, T. M., Anderson, J. L., Bush, S. R., Smith, M. D., Anderson, C. M., ... Oglend, A. (2018). Three pillars of sustainability in fisheries. Proceedings of the National Academy of Sciences, 115(44), 1122111225.

Auger, K. A., Shah, S. S., Richardson, T., Hartley, D., Hall, M., Warniment, A., ... Schondelmeyer, A. C. (2020). Association between statewide school closure and COVID-19 incidence and mortality in the US. Jama, 324(9), 859-870.

Banco de España. (2020). Escenarios macroeconómicos de referencia para la economía española tras el Covid19. ARTÍCULOS ANALÍTICOS Boletín Económico, 2, 33.

Belzunegui-Eraso, A., \& Erro-Garcés, A. (2020). Teleworking in the context of the Covid-19 crisis. Sustainability (Switzerland), 12(9), 1-18. https://doi.org/10.3390/su12093662

Bland, J. M., \& Altman, D. G. (1997). Statistics notes: Cronbach's alpha. Bmj, 314(7080), 572.

Bosoer, F., \& Turzi, M. (2020). La pandemia del 2020 en el debate teórico de las Relaciones Internacionales. Geopolitica, 11, 153.

Brencht, B. (1997). (1997, p. 290),. 290. 
Brown, B. J., Hanson, M. E., Liverman, D. M., \& Merideth, R. W. (1987). Global sustainability: Toward definition. Environmental Management, 11(6), 713-719. https://doi.org/10.1007/BF01867238

Cadil, J., Mirosnik, K., Petkovova, L., \& Mirvald, M. (2018). Public Support of Private R\&D-Effects on Economic Sustainability. Sustainability, 10(12), 4612.

Christen, M., \& Schmidt, S. (2012). A formal framework for conceptions of sustainability-a theoretical contribution to the discourse in sustainable development. Sustainable Development, 20(6), 400-410.

Dagiliūtè, R., Liobikienè, G., \& Minelgaitè, A. (2018). Sustainability at universities: Students' perceptions from Green and Non-Green universities. Journal of Cleaner Production, 181, 473-482.

de Las Heras-Pedrosa, C., Sánchez-Núñez, P., \& Peláez, J. I. (2020). Sentiment Analysis and Emotion Understanding during the COVID-19 Pandemic in Spain and Its Impact on Digital Ecosystems. International Journal of Environmental Research and Public Health, 17(15), 5542.

Del Sordo, C., Farneti, F., Guthrie, J., Pazzi, S., \& Siboni, B. (2016). Social reports in Italian universities: disclosures and preparers' perspective. Meditari Accountancy Research.

Dempsey, N., Bramley, G., Power, S., \& Brown, C. (2011). The social dimension of sustainable development: Defining urban social sustainability. Sustainable Development, 19(5), 289-300. https://doi.org/10.1002/sd.417

Dima, A. M., Țuclea, C. E., Vrânceanu, D. M., \& Țigu, G. (2019). Sustainable Social and Individual Implications of Telework: A New Insight into the Romanian Labor Market. Sustainability, 11(13), 3506.

Eizenberg, E., \& Jabareen, Y. (2017). Social sustainability: A new conceptual framework. Sustainability, 9(1), 68.

Esnaashari, M., \& Sarvari, H. (2020). A survey of factors affecting the citizen's behaviors in Mashhad, Iran. Environment, Development and Sustainability, 1(21).

García-Peñalvo, F. J., Corell, A., Abella-García, V., \& Grande, M. (2020). La evaluación online en la educación superior en tiempos de la COVID-19. Education in the Knowledge Society (EKS), 21(0), 26. https://doi.org/10.14201/eks.23086

Geissdoerfer, M., Savaget, P., Bocken, N. M. P., \& Hultink, E. J. (2019). Circular Economy Sustainability Paradigm. Journal of Cleaner Production, 143(June), 757-768. https://doi.org/10.1016/j.jclepro.2016.12.048.Abstract

Goodland, R. (1998). The urgent need for ecosystem integrity and ethics to support environmental sustainability. Global Bioethics, 11(1-4), 29-46. https://doi.org/10.1080/11287462.1998.10800729

Hák, T., Janouškova, S., Moldan, B., \& Dahl, A. L. (2018). Closing the sustainability gap: 30 years after "Our Common Future", society lacks meaningful stories and relevant indicators to make the right decisions and build public support. Ecological Indicators, 87, 193-195.

Hodges, C., Moore, S., Lockee, B., Trust, T., \& Bond, A. (2020). The difference between emergency remote teaching and online learning. Educause Review.

Howard, M. C. (2016). A review of exploratory factor analysis decisions and overview of current practices: What we are doing and how can we improve?. International Journal of Human-Computer Interaction, 32(1), 5162. 
Huerta-Riveros, P., \& Gaete-Feres, H. (2017). Responsabilidad social universitaria a través de los reportes de sostenibilidad del Global Reporting Initiative: experiencia de una universidad pública. Revista Iberoamericana de Educación Superior, 8(23), 120-137.

Isman, A., \& Canan Gungoren, O. (2014). Digital citizenship. Turkish Online Journal of Educational TechnologyTOJET, 13(1), 73-77.

Janoušková, S., Hák, T., \& Moldan, B. (2018). Global SDGs assessments: Helping or confusing indicators? Sustainability, 10(5), 1540.

Karji, A., Woldesenbet, A., Khanzadi, M., \& Tafazzoli, M. (2019). Assessment of Social Sustainability Indicators in Mass Housing Construction: A Case Study of Mehr Housing Project. Sustainable Cities and Society. (50), 101697.

Lal, P., Kumar, A., Kumar, S., Kumari, S., Saikia, P., Dayanandan, A., ... Khan, M. L. (2020). The dark cloud with a silver lining: Assessing the impact of the SARS COVID-19 pandemic on the global environment. ,. Science of the Total Environment, 732, 139297.

Larrán-Jorge, M., \& Andrades-Peña, F. J. (2015). Análisis de la responsabilidad social universitaria desde diferentes enfoques teóricos. Revista Iberoamericana de Educación Superior, 6(15), 91-107.

Leal Filho, W., Brandli, L. L., Lange Salvia, A., Rayman-Bacchus, L., \& Platje, J. (2020). COVID-19 and the UN sustainable development goals: threat to solidarity or an opportunity?. Sustainability, 12(13), 5343.

Lozano, R., Ceulemans, K., Alonso-Almeida, M., Huisingh, D., Lozano, F. J., Waas, T., ... Hugé, J. (2015). A review of commitment and implementation of sustainable development in higher education: results from a worldwide survey. Journal of Cleaner Production, (108), 1-18.

Martínez-Navalón, J. G., Gelashvili, V., \& Debasa, F. (2019). The impact of restaurant social media on environmental sustainability: An empirical study. Sustainability (Switzerland), 11(21). https://doi.org/10.3390/su11216105

Messenger, J. C., \& Gschwind, L. (2016). Three generations of Telework: New ICT s and the (R) evolution from Home Office to Virtual Office. New Technology, Work and Employment, 31(3), 195-208.

Moldan, B., Janoušková, S., \& Hák, T. (2012). How to understand and measure environmental sustainability: Indicators and targets. Ecological Indicators, 17, 4-13. https://doi.org/10.1016/j.ecolind.2011.04.033

Moos, M., Andrey, J., \& Johnson, L. C. (2017). The sustainability of telework: an ecological-footprinting approach. Sustainability: Science, Practice and Policy, 2(1), 3-14.

Muñoz, V. G., Callejo, M. R. S., Sastre, L. B., \& Marín, A. C. (2017). Revisión sistemática sobre competencias en desarrollo sostenible en educación superior. Revista Iberoamericana de Educación, 73(4), 34-56.

Nakrošienè, A., Bučiūnienè, I., \& Goštautaitè, B. (2019). Working from home: characteristics and outcomes of telework. International Journal of Manpower, 40(1), 87-101. https://doi.org/10.1108/IJM-07-2017-0172

Narayanan, L., Menon, S., Plaisent, M., \& Bernard, P. (2017). Telecommuting: The work anywhere, anyplace, anytime organization in the 21st century. Journal of Marketing \& Management, 8(2), 47-54.

Nicola, M., Alsafi, Z., Sohrabi, C., Kerwan, A., Al-Jabir, A., losifidis, C., \& ... Agha, R. (2020). The socio-economic implications of the coronavirus pandemic (COVID-19): A review. International Journal of Surgery (London, England), 78, 185. 
Ong, M. H. A., \& Puteh, F. (2017). Quantitative data analysis: Choosing between SPSS, PLS and AMOS in social science research. International Interdisciplinary Journal of Scientific Research, 3(1), 14-25.

Pena-Cerezo, M. A., Artaraz-Minon, M., \& Tejedor-Nunez, J. (2019). Analysis of the Consciousness of University Undergraduates for Sustainable Consumption. Sustainability, 11(17), 4597.

Purvis, B., Mao, Y., \& Robinson, D. (2019). Three pillars of sustainability: in search of conceptual origins. Sustainability Science, 14(3), 681-695.

Reyes-Menendez, A., Saura, J. R., \& Martinez-Navalon, J. G. (2019). The Impact of e-WOM on Hotels Management Reputation: Exploring TripAdvisor Review Credibility with the ELM Model. IEEE Access, 7, 68868-68877. https://doi.org/10.1109/ACCESS.2019.2919030

Rogge, N., Theesfeld, I., \& Strassner, C. (2018). Social sustainability through social interaction-A national survey on community gardens in Germany. 10(4), 1085.

Sandín, B., Valiente, R. M., García-Escalera, J., \& Chorot, P. (2020). Impacto psicológico de la pandemia de COVID-19: Efectos negativos y positivos en población española asociados al periodo de confinamiento nacional. Revista de Psicopatologia y Psicologia Clinica, 25(1).

Sierra, M. A. (2020). Infección respiratoria aguda por COVID-19: una amenaza evidente. Revista Habanera De Ciencias Medicas, 6(5), 1-15. Retrieved from http://scielo.sld.cu/scielo.php?pid=S1729519X2008000300003\&script=sci_arttext

Singh Niranjan, P. (2020). "Coronavirus Pandemic impact on Global Education: A Blessing in disguise." Sustainable Humanosphere, 16(2), 68-72.

Thabiso, M., \& Awuzie, B. (2017). Mainstreaming Social Sustainability into Infrastructure Delivery Systems: Are There Any Benefits? A Stakeholders' Perspective. 11th Built Environment Conference.

Ukko, J., Saunila, M., Rantala, T., \& Havukainen, J. (2019). Sustainable development: Implications and definition for open sustainability. Sustainable Development.

World Comission on Environment and Development. (1987). Our common future. Oxford University Press.

\section{Anexos}

Cuadro Anexo 1

Cuadro de equivalencias : variable-pregunta

\begin{tabular}{llllll}
\hline 1 & Género & Género & 30 & C.online-perf-2 & $\begin{array}{l}\text { Cree que el futuro será } \\
\text { mejor online }\end{array}$ \\
\hline 2 & Edad & Edad & 31 & C.online-perf-3 & $\begin{array}{l}\text { Aprendes mejor que con el } \\
\text { sistema presencial }\end{array}$ \\
\hline 3 & Universidad & ¿En qué universidad estudia? & 32 & C.online-capa-1 & $\begin{array}{l}\text { Me he adaptado muy rápido } \\
\text { a tener que usar nuevas } \\
\text { tecnologías }\end{array}$ \\
\hline 5 & Covid-gene-1 & $\begin{array}{l}\text { He experimentado cambios a } \\
\text { raíz del COVID-19 }\end{array}$ & 33 & C.online-capa-2 & $\begin{array}{l}\text { La flexibilidad de las clases } \\
\text { online me permite } \\
\text { aumentar la productividad }\end{array}$ \\
\hline
\end{tabular}




\begin{tabular}{|c|c|c|c|c|c|}
\hline 6 & Covid-gene-2 & $\begin{array}{l}\text { Me he sentido menos capaz de } \\
\text { hacer mis tareas porque mi } \\
\text { propio bienestar ha sufrido el } \\
\text { estrés de la pandemia }\end{array}$ & 34 & C.online-capa-3 & $\begin{array}{l}\text { La flexibilidad de las clases } \\
\text { online me permite tener } \\
\text { menos estrés que antes }\end{array}$ \\
\hline 7 & Covid-gene-3 & $\begin{array}{l}\text { He tenido mayor dificultad para } \\
\text { lograr el equilibrio entre las } \\
\text { clases y la vida privada }\end{array}$ & 35 & C.online-herra-1 & $\begin{array}{l}\text { Las comunicaciones } \\
\text { institucionales son } \\
\text { altamente efectivos }\end{array}$ \\
\hline 8 & Covid-convi-1 & $\begin{array}{l}\text { ¿Con quién ha pasado el periodo } \\
\text { de confinamiento derivado del } \\
\text { COVID-19? }\end{array}$ & 36 & C.online-herra-2 & $\begin{array}{l}\text { El correo electrónico } \\
\text { institucional cumple con el } \\
\text { envío y recepción de } \\
\text { información }\end{array}$ \\
\hline 9 & Covid-convi-2 & $\begin{array}{l}\text { ¿Ha convivido durante este } \\
\text { periodo con algún niño menor } \\
\text { de } 18 \text { años? }\end{array}$ & 37 & C.online-herra-3 & $\begin{array}{l}\text { Tengo pleno acceso al aula } \\
\text { virtual desde mi residencia }\end{array}$ \\
\hline 10 & Covid-enferm-1 & $\begin{array}{l}\text { ¿Ha convivido durante este } \\
\text { periodo con personas mayores o } \\
\text { discapacitados? }\end{array}$ & 38 & C.online-condi-1 & $\begin{array}{l}\text { Tengo espacio individual y } \\
\text { privado para el estudio }\end{array}$ \\
\hline 11 & Covid-enferm-2 & $\begin{array}{l}\text { ¿Cree que ha tenido una } \\
\text { infección por COVID-19? }\end{array}$ & 39 & C.online-condi-2 & $\begin{array}{l}\text { Tengo ordenador propio } \\
\text { para el estudio }\end{array}$ \\
\hline 12 & Covid-enferm-3 & $\begin{array}{l}\text { ¿Cree que alguien en su casa ha } \\
\text { tenido una infección por COVID- } \\
19 ?\end{array}$ & 40 & C.online-condi-3 & $\begin{array}{l}\text { Tengo conexión a internet } \\
\text { banda ancha (wifi, cable o } \\
\text { ADSL) }\end{array}$ \\
\hline 13 & Covid-emoci-1 & $\begin{array}{l}\text { ¿Cuánto ha afectado el } \\
\text { confinamiento a tu estado } \\
\text { emocional? }\end{array}$ & 41 & C.online-psico-1 & $\begin{array}{l}\text { Creo que me distraería con } \\
\text { facilidad si fuera modalidad } \\
\text { online }\end{array}$ \\
\hline 14 & Covid-rendim-1 & $\begin{array}{l}\text { Tu rendimiento académico en } \\
\text { los estudios universitarios } \\
\text { actualmente es }\end{array}$ & 42 & C.online-psico-2 & $\begin{array}{l}\text { Tendría mayor atención a } \\
\text { sus responsabilidades y me } \\
\text { rendiría más el tiempo bajo } \\
\text { la modalidad online }\end{array}$ \\
\hline 15 & Covid-rendim-2 & $\begin{array}{l}\text { Indica la respuesta que mejor } \\
\text { reflejaba tu rendimiento } \\
\text { antes del COVID-9 }\end{array}$ & 43 & C.online-psico-3 & $\begin{array}{l}\text { Me he sentido presionado } \\
\text { por la cantidad de carga de } \\
\text { trabajo }\end{array}$ \\
\hline 17 & Sost-gen-1 & $\begin{array}{l}\text { Me interesan los temas } \\
\text { relacionados con la } \\
\text { sostenibilidad }\end{array}$ & 44 & C.online-cult-1 & $\begin{array}{l}\text { Me hace falta ir a la } \\
\text { Universidad }\end{array}$ \\
\hline 18 & Sost-gen-2 & $\begin{array}{l}\text { Me considero una persona } \\
\text { sostenible }\end{array}$ & 45 & C.online-cult-2 & $\begin{array}{l}\text { Conozco las ventajas de las } \\
\text { clases online }\end{array}$ \\
\hline 19 & Sost-gen-3 & $\begin{array}{l}\text { Considero que es importante } \\
\text { que organizaciones como la } \\
\text { educación sean sostenibles }\end{array}$ & 46 & C.online-cult-3 & $\begin{array}{l}\text { Conozco los aspectos legales } \\
\text { de las clases online }\end{array}$ \\
\hline 20 & Sost- soci-1 & $\begin{array}{l}\text { La universidad en la que estudia } \\
\text { ayuda a la mejora de la calidad } \\
\text { de vida de la comunidad. }\end{array}$ & 47 & C.online-comu-1 & $\begin{array}{l}\text { Tengo dificultades para } \\
\text { estudiar el programa } \\
\text { establecido }\end{array}$ \\
\hline 21 & Sost- soci-2 & $\begin{array}{l}\text { La universidad en la que estudia } \\
\text { ayuda a resolver problemas } \\
\text { sociales }\end{array}$ & 48 & C.online-comu-2 & $\begin{array}{l}\text { Me resulta complicado } \\
\text { atender en las clases online }\end{array}$ \\
\hline
\end{tabular}




\section{Sost- soci-3}

La universidad en la que estudia brinda un trato justo a os empleados (no discrimina)

La universidad en la que estudia

23 Sost- eco-1 trata de hacer todo lo posible por ser productiva

La universidad en la que estudia intenta mejorar continuamente la calidad de los servicios que ofrece

La universidad en la que estudia intenta construir relaciones a

25 Sost- eco-3 largo plazo con sus grupos de interés para asegurarse su éxito a largo plazo
Tengo problemas para

comprender los nuevos

49 C.online-comu-3 temas o contenidos de la asignatura

Si accediera a la modalidad

50 C.online-fami-1 online, sería inevitable que mi familia me interrumpiera

Si accediera a la modalidad

51 C.online-fami-2 online, el ahorro de tiempo en desplazamientos lo dedicaría a mi familia y a mí

Si accediera a la modalidad

52 C.online-fami-3 de clases online, mejorarían mis relaciones familiares

Considero que las

La universidad en la que estudia

26 Sost- med-1 valora y protege el medioambiente

53 C.online-sost-1

universidades españolas

aportan a la sostenibilidad

incentivando esta

modalidad de aprendizaje

Considero que la modalidad

La universidad en la que estudia

27 Sost- med-2 desarrolla políticas activas de reciclaje

54 C.online-sost-2

de clases online ha contribuido a mejorar mi desempeño académico

Sé valorar el impacto (positivo y negativo) de las

La universidad en la que estudia

28 Sost- med-3 tiene campañas de concienciación contra la

55 C.online-sost-3 clases online en la sociedad y en la sostenibilidad del planeta

Me gustaría que estos cambios

29 C.online-perf-1 se mantuviesen aun cuando pase el COVID-19
56 C.online-gene-1 prefiero estudiar
Haciendo balance general,

\section{Fuente de elaboración propia}

Cuadro Anexo 2

Resultados de la encuesta de los bloques 2, 3 y 4

\begin{tabular}{|c|c|c|c|c|}
\hline & Clasificación & Variable & $\mathrm{fr}$ & $\%$ \\
\hline \multirow{5}{*}{5} & \multirow{5}{*}{ Covid-gene-1 } & 1 & 5 & $1 \%$ \\
\hline & & 2 & 11 & $3 \%$ \\
\hline & & 3 & 26 & $6 \%$ \\
\hline & & 4 & 222 & $54 \%$ \\
\hline & & 5 & 147 & $36 \%$ \\
\hline \multirow{4}{*}{6} & \multirow{4}{*}{ Covid-gene-2 } & 1 & 25 & $6 \%$ \\
\hline & & 2 & 58 & $14 \%$ \\
\hline & & 3 & 63 & $15 \%$ \\
\hline & & 4 & 184 & $45 \%$ \\
\hline
\end{tabular}

\begin{tabular}{|c|c|c|c|c|}
\hline & Clasificación & Variable & $\mathrm{fr}$ & $\%$ \\
\hline & & 5 & 81 & $20 \%$ \\
\hline \multirow{5}{*}{7} & \multirow{5}{*}{ Covid-gene-3 } & 1 & 24 & $6 \%$ \\
\hline & & 2 & 73 & $18 \%$ \\
\hline & & 3 & 61 & $15 \%$ \\
\hline & & 4 & 138 & $34 \%$ \\
\hline & & 5 & 115 & $28 \%$ \\
\hline \multirow{3}{*}{8} & \multirow{3}{*}{ Covid-convi-1 } & 1 & 5 & $1 \%$ \\
\hline & & 2 & 9 & $2 \%$ \\
\hline & & 3 & 29 & $7 \%$ \\
\hline
\end{tabular}




\begin{tabular}{|c|c|c|c|c|}
\hline & Clasificación & Variable & $\mathrm{fr}$ & $\%$ \\
\hline & & 4 & 10 & $2 \%$ \\
\hline & & 5 & 1 & $0 \%$ \\
\hline & & 6 & 357 & $87 \%$ \\
\hline \multirow{2}{*}{9} & \multirow{2}{*}{ Covid-convi-2 } & 1 & 126 & $31 \%$ \\
\hline & & 2 & 285 & $69 \%$ \\
\hline \multirow{2}{*}{10} & \multirow{2}{*}{ Covid-enferm-1 } & 1 & 56 & $14 \%$ \\
\hline & & 2 & 355 & $86 \%$ \\
\hline \multirow{3}{*}{11} & \multirow{3}{*}{ Covid-enferm-2 } & 1 & 17 & $4 \%$ \\
\hline & & 2 & 79 & $19 \%$ \\
\hline & & 3 & 315 & $77 \%$ \\
\hline \multirow{3}{*}{12} & \multirow{3}{*}{ Covid-enferm-3 } & 1 & 40 & $10 \%$ \\
\hline & & 2 & 83 & $20 \%$ \\
\hline & & 3 & 288 & $70 \%$ \\
\hline \multirow{4}{*}{13} & \multirow{4}{*}{ Covid-emoci-1 } & 1 & 21 & $5 \%$ \\
\hline & & 2 & 152 & $37 \%$ \\
\hline & & 3 & 175 & $43 \%$ \\
\hline & & 4 & 63 & $15 \%$ \\
\hline \multirow{5}{*}{14} & \multirow{5}{*}{ Covid-rendim-1 } & 1 & 9 & $2 \%$ \\
\hline & & 2 & 54 & $13 \%$ \\
\hline & & 3 & 118 & $29 \%$ \\
\hline & & 4 & 162 & $39 \%$ \\
\hline & & 5 & 68 & $17 \%$ \\
\hline \multirow{4}{*}{15} & \multirow{4}{*}{ Covid-rendim-2 } & 1 & 109 & $27 \%$ \\
\hline & & 2 & 177 & $43 \%$ \\
\hline & & 3 & 98 & $24 \%$ \\
\hline & & 4 & 27 & $7 \%$ \\
\hline \multirow{5}{*}{17} & \multirow{5}{*}{ Sost-gen-1 } & 1 & 8 & $2 \%$ \\
\hline & & 2 & 13 & $3 \%$ \\
\hline & & 3 & 74 & $18 \%$ \\
\hline & & 4 & 195 & $47 \%$ \\
\hline & & 5 & 121 & $29 \%$ \\
\hline \multirow{5}{*}{18} & \multirow{5}{*}{ Sost-gen-2 } & 1 & 2 & $0 \%$ \\
\hline & & 2 & 22 & $5 \%$ \\
\hline & & 3 & 115 & $28 \%$ \\
\hline & & 4 & 234 & $57 \%$ \\
\hline & & 5 & 38 & $9 \%$ \\
\hline \multirow{4}{*}{19} & \multirow{4}{*}{ Sost-gen-3 } & 1 & 5 & $1 \%$ \\
\hline & & 2 & 4 & $1 \%$ \\
\hline & & 3 & 22 & $5 \%$ \\
\hline & & 4 & 152 & $37 \%$ \\
\hline
\end{tabular}

\begin{tabular}{|c|c|c|c|c|}
\hline & Clasificación & Variable & $\mathrm{fr}$ & $\%$ \\
\hline & & 5 & 228 & $55 \%$ \\
\hline \multirow{5}{*}{20} & \multirow{5}{*}{ Sost- soci-1 } & 1 & 11 & $3 \%$ \\
\hline & & 2 & 58 & $14 \%$ \\
\hline & & 3 & 177 & $43 \%$ \\
\hline & & 4 & 133 & $32 \%$ \\
\hline & & 5 & 32 & $8 \%$ \\
\hline \multirow{5}{*}{21} & \multirow{5}{*}{ Sost- soci-2 } & 1 & 16 & $4 \%$ \\
\hline & & 2 & 72 & $18 \%$ \\
\hline & & 3 & 182 & $44 \%$ \\
\hline & & 4 & 122 & $30 \%$ \\
\hline & & 5 & 19 & $5 \%$ \\
\hline \multirow{5}{*}{22} & \multirow{5}{*}{ Sost- soci-3 } & 1 & 8 & $2 \%$ \\
\hline & & 2 & 37 & $9 \%$ \\
\hline & & 3 & 168 & $41 \%$ \\
\hline & & 4 & 153 & $37 \%$ \\
\hline & & 5 & 45 & $11 \%$ \\
\hline \multirow{5}{*}{23} & \multirow{5}{*}{ Sost- eco-1 } & 1 & 18 & $4 \%$ \\
\hline & & 2 & 58 & $14 \%$ \\
\hline & & 3 & 131 & $32 \%$ \\
\hline & & 4 & 179 & $44 \%$ \\
\hline & & 5 & 25 & $6 \%$ \\
\hline \multirow{5}{*}{24} & \multirow{5}{*}{ Sost- eco-2 } & 1 & 33 & $8 \%$ \\
\hline & & 2 & 76 & $18 \%$ \\
\hline & & 3 & 122 & $30 \%$ \\
\hline & & 4 & 150 & $36 \%$ \\
\hline & & 5 & 30 & $7 \%$ \\
\hline \multirow{5}{*}{25} & \multirow{5}{*}{ Sost- eco-3 } & 1 & 21 & $5 \%$ \\
\hline & & 2 & 73 & $18 \%$ \\
\hline & & 3 & 164 & $40 \%$ \\
\hline & & 4 & 128 & $31 \%$ \\
\hline & & 5 & 25 & $6 \%$ \\
\hline \multirow{5}{*}{26} & \multirow{5}{*}{ Sost- med-1 } & 1 & 9 & $2 \%$ \\
\hline & & 2 & 39 & $9 \%$ \\
\hline & & 3 & 142 & $35 \%$ \\
\hline & & 4 & 182 & $44 \%$ \\
\hline & & 5 & 39 & $9 \%$ \\
\hline \multirow{4}{*}{27} & \multirow{4}{*}{ Sost- med-2 } & 1 & 15 & $4 \%$ \\
\hline & & 2 & 55 & $13 \%$ \\
\hline & & 3 & 152 & $37 \%$ \\
\hline & & 4 & 147 & $36 \%$ \\
\hline
\end{tabular}




\begin{tabular}{|c|c|c|c|c|}
\hline & Clasificación & Variable & fr & $\%$ \\
\hline & & 5 & 42 & $10 \%$ \\
\hline \multirow{5}{*}{28} & \multirow{5}{*}{ Sost- med-3 } & 1 & 20 & $5 \%$ \\
\hline & & 2 & 69 & $17 \%$ \\
\hline & & 3 & 181 & $44 \%$ \\
\hline & & 4 & 115 & $28 \%$ \\
\hline & & 5 & 26 & $6 \%$ \\
\hline \multirow{5}{*}{29} & \multirow{5}{*}{ C.online-perf-1 } & 1 & 75 & $18 \%$ \\
\hline & & 2 & 93 & $23 \%$ \\
\hline & & 3 & 146 & $36 \%$ \\
\hline & & 4 & 78 & $19 \%$ \\
\hline & & 5 & 19 & $5 \%$ \\
\hline \multirow{5}{*}{30} & \multirow{5}{*}{ C.online-perf-2 } & 1 & 136 & $33 \%$ \\
\hline & & 2 & 127 & $31 \%$ \\
\hline & & 3 & 103 & $25 \%$ \\
\hline & & 4 & 34 & $8 \%$ \\
\hline & & 5 & 11 & $3 \%$ \\
\hline \multirow{5}{*}{31} & \multirow{5}{*}{ C.online-perf-3 } & 1 & 116 & $28 \%$ \\
\hline & & 2 & 83 & $20 \%$ \\
\hline & & 3 & 56 & $14 \%$ \\
\hline & & 4 & 62 & $15 \%$ \\
\hline & & 5 & 94 & $23 \%$ \\
\hline \multirow{5}{*}{32} & \multirow{5}{*}{ C.online-capa-1 } & 1 & 11 & $3 \%$ \\
\hline & & 2 & 35 & $9 \%$ \\
\hline & & 3 & 77 & $19 \%$ \\
\hline & & 4 & 186 & $45 \%$ \\
\hline & & 5 & 102 & $25 \%$ \\
\hline \multirow{5}{*}{33} & \multirow{5}{*}{ C.online-capa-2 } & 1 & 55 & $13 \%$ \\
\hline & & 2 & 115 & $28 \%$ \\
\hline & & 3 & 93 & $23 \%$ \\
\hline & & 4 & 104 & $25 \%$ \\
\hline & & 5 & 44 & $11 \%$ \\
\hline \multirow{5}{*}{34} & \multirow{5}{*}{ C.online-capa-3 } & 1 & 71 & $17 \%$ \\
\hline & & 2 & 130 & $32 \%$ \\
\hline & & 3 & 100 & $24 \%$ \\
\hline & & 4 & 79 & $19 \%$ \\
\hline & & 5 & 31 & $8 \%$ \\
\hline \multirow{4}{*}{35} & \multirow{4}{*}{ C.online-herra-1 } & 1 & 20 & $5 \%$ \\
\hline & & 2 & 67 & $16 \%$ \\
\hline & & 3 & 167 & $41 \%$ \\
\hline & & 4 & 136 & $33 \%$ \\
\hline
\end{tabular}

\begin{tabular}{|c|c|c|c|c|}
\hline & Clasificación & Variable & fr & $\%$ \\
\hline & & 5 & 21 & $5 \%$ \\
\hline \multirow{5}{*}{36} & \multirow{5}{*}{ C.online-herra-2 } & 1 & 9 & $2 \%$ \\
\hline & & 2 & 58 & $14 \%$ \\
\hline & & 3 & 93 & $23 \%$ \\
\hline & & 4 & 178 & $43 \%$ \\
\hline & & 5 & 73 & $18 \%$ \\
\hline \multirow{5}{*}{37} & \multirow{5}{*}{ C.online-herra-3 } & 1 & 1 & $0 \%$ \\
\hline & & 2 & 3 & $1 \%$ \\
\hline & & 3 & 25 & $6 \%$ \\
\hline & & 4 & 81 & $20 \%$ \\
\hline & & 5 & 301 & $73 \%$ \\
\hline \multirow{5}{*}{38} & \multirow{5}{*}{ C.online-condi-1 } & 1 & 16 & $4 \%$ \\
\hline & & 2 & 25 & $6 \%$ \\
\hline & & 3 & 38 & $9 \%$ \\
\hline & & 4 & 108 & $26 \%$ \\
\hline & & 5 & 224 & $55 \%$ \\
\hline \multirow{5}{*}{39} & \multirow{5}{*}{ C.online-condi-2 } & 1 & 8 & $2 \%$ \\
\hline & & 2 & 12 & $3 \%$ \\
\hline & & 3 & 15 & $4 \%$ \\
\hline & & 4 & 46 & $11 \%$ \\
\hline & & 5 & 330 & $80 \%$ \\
\hline \multirow{5}{*}{40} & \multirow{5}{*}{ C.online-condi-3 } & 1 & 2 & $0 \%$ \\
\hline & & 2 & 8 & $2 \%$ \\
\hline & & 3 & 20 & $5 \%$ \\
\hline & & 4 & 75 & $18 \%$ \\
\hline & & 5 & 306 & $74 \%$ \\
\hline \multirow{5}{*}{41} & \multirow{5}{*}{ C.online-psico-1 } & 1 & 18 & $4 \%$ \\
\hline & & 2 & 38 & $9 \%$ \\
\hline & & 3 & 84 & $20 \%$ \\
\hline & & 4 & 162 & $39 \%$ \\
\hline & & 5 & 109 & $27 \%$ \\
\hline \multirow{5}{*}{42} & \multirow{5}{*}{ C.online-psico-2 } & 1 & 84 & $20 \%$ \\
\hline & & 2 & 142 & $35 \%$ \\
\hline & & 3 & 107 & $26 \%$ \\
\hline & & 4 & 50 & $12 \%$ \\
\hline & & 5 & 28 & $7 \%$ \\
\hline \multirow{4}{*}{43} & \multirow{4}{*}{ C.online-psico-3 } & 1 & 17 & $4 \%$ \\
\hline & & 2 & 36 & $9 \%$ \\
\hline & & 3 & 98 & $24 \%$ \\
\hline & & 4 & 118 & $29 \%$ \\
\hline
\end{tabular}




\begin{tabular}{|c|c|c|c|c|c|c|c|c|c|}
\hline & Clasificación & Variable & fr & $\%$ & & Clasificación & Variable & fr & $\%$ \\
\hline & & 5 & 142 & $35 \%$ & & & 2 & 90 & $22 \%$ \\
\hline \multirow{5}{*}{44} & \multirow{5}{*}{ C.online-cult-1 } & 1 & 15 & $4 \%$ & & & 3 & 76 & $18 \%$ \\
\hline & & 2 & 32 & $8 \%$ & & & 4 & 130 & $32 \%$ \\
\hline & & 3 & 61 & $15 \%$ & & & 5 & 66 & $16 \%$ \\
\hline & & 4 & 162 & $39 \%$ & \multirow{5}{*}{51} & \multirow{5}{*}{ C.online-fami-2 } & 1 & 19 & $5 \%$ \\
\hline & & 5 & 141 & $34 \%$ & & & 2 & 49 & $12 \%$ \\
\hline \multirow{5}{*}{45} & \multirow{5}{*}{ C.online-cult-2 } & 1 & 15 & $4 \%$ & & & 3 & 83 & $20 \%$ \\
\hline & & 2 & 38 & $9 \%$ & & & 4 & 160 & $39 \%$ \\
\hline & & 3 & 85 & $21 \%$ & & & 5 & 100 & $24 \%$ \\
\hline & & 4 & 211 & $51 \%$ & \multirow{5}{*}{52} & \multirow{5}{*}{ C.online-fami-3 } & 1 & 51 & $12 \%$ \\
\hline & & 5 & 62 & $15 \%$ & & & 2 & 118 & $29 \%$ \\
\hline \multirow{5}{*}{46} & \multirow{5}{*}{ C.online-cult-3 } & 1 & 71 & $17 \%$ & & & 3 & 160 & $39 \%$ \\
\hline & & 2 & 121 & $29 \%$ & & & 4 & 52 & $13 \%$ \\
\hline & & 3 & 91 & $22 \%$ & & & 5 & 30 & $7 \%$ \\
\hline & & 4 & 104 & $25 \%$ & \multirow{5}{*}{53} & \multirow{5}{*}{ C.online-sost-1 } & 1 & 38 & $9 \%$ \\
\hline & & 5 & 24 & $6 \%$ & & & 2 & 94 & $23 \%$ \\
\hline \multirow{5}{*}{47} & \multirow{5}{*}{ C.online-comu-1 } & 1 & 24 & $6 \%$ & & & 3 & 189 & $46 \%$ \\
\hline & & 2 & 129 & $31 \%$ & & & 4 & 79 & $19 \%$ \\
\hline & & 3 & 191 & $46 \%$ & & & 5 & 11 & $3 \%$ \\
\hline & & 4 & 57 & $14 \%$ & \multirow{5}{*}{54} & \multirow{5}{*}{ C.online-sost-2 } & 1 & 75 & $18 \%$ \\
\hline & & 5 & 10 & $2 \%$ & & & 2 & 128 & $31 \%$ \\
\hline \multirow{5}{*}{48} & \multirow{5}{*}{ C.online-comu-2 } & 1 & 25 & $6 \%$ & & & 3 & 111 & $27 \%$ \\
\hline & & 2 & 63 & $15 \%$ & & & 4 & 76 & $18 \%$ \\
\hline & & 3 & 131 & $32 \%$ & & & 5 & 21 & $5 \%$ \\
\hline & & 4 & 124 & $30 \%$ & \multirow{5}{*}{55} & \multirow{5}{*}{ C.online-sost-3 } & 1 & 18 & $4 \%$ \\
\hline & & 5 & 68 & $17 \%$ & & & 2 & 30 & $7 \%$ \\
\hline \multirow{5}{*}{49} & \multirow{5}{*}{ C.online-comu-3 } & 1 & 30 & $7 \%$ & & & 3 & 98 & $24 \%$ \\
\hline & & 2 & 130 & $32 \%$ & & & 4 & 215 & $52 \%$ \\
\hline & & 3 & 153 & $37 \%$ & & & 5 & 50 & $12 \%$ \\
\hline & & 4 & 76 & $18 \%$ & \multirow{3}{*}{56} & \multirow{3}{*}{ C.online-gene-1 } & 1 & 215 & $52 \%$ \\
\hline & & 5 & 22 & $5 \%$ & & & 2 & 27 & $7 \%$ \\
\hline 50 & C.online-fami-1 & 1 & 49 & $12 \%$ & & & 3 & 169 & $41 \%$ \\
\hline
\end{tabular}

Fuente de elaboración propia

Esta obra está bajo una Licencia Creative Commons

Attribución-NoCommercial 4.0 International

(cc) BY-NC 